\title{
Tracking Time-varying Systems: Revisiting Some Basic Trade-offs
}

\author{
Urban Forssell and Lennart Ljung \\ Department of Electrical Engineering \\ Linköping University, S-581 83 Linköping, Sweden \\ www: http://www.control.isy.liu.se \\ email: ufo@isy.liu.se, ljung@isy.liu.se
}

1997-04-10

Conference: SYSID '97

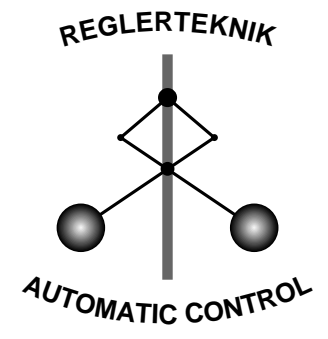

LINKÖPING

Technical reports from the Automatic Control group in Linköping are available by anonymous ftp at the address 130.236.20.24 (ftp.control.isy.liu.se). This report is contained in the compressed postscript file LiTH-ISY-R-1944.ps.Z. 


\title{
TRACKING TIME-VARYING SYSTEMS: REVISITING SOME BASIC TRADE-OFFS
}

\author{
U. Forssell and L. Ljung \\ Department of Electrical Engineering, Linköping University, \\ Linköping, SWEDEN. \\ E-mail: ufoisy.liu.se, ljungisy.liu.se
}

\begin{abstract}
The trade-off between tracking ability and noise sensitivity in adaptive algorithms is a classical research topic. In this paper we will revisit some of the main results. An input/output view of the archetypical algorithms for recursive identification will be taken, thus enabling the links to $\mathcal{H}_{\infty}$ and $\mathcal{H}_{2}$ norms. We will shed some light on the $\mathcal{H}_{\infty}$ optimality of the LMS algorithm and also show the corresponding merits of the RLS-type algorithms. Furthermore, the role of the distribution of the noise will be illustrated.
\end{abstract}

Keywords: Recursive identification; $\mathcal{H}_{\infty}$ estimation; Kalman filter; RLS; LMS.

\section{INTRODUCTION: MEASURES OF TRACKING ABILITY}

The basic problem of tracking a possibly timevarying linear regression parameter vector has been treated extensively in the literature. See, e.g., (Ljung and Söderström, 1983) or (Widrow and Stearns, 1985). The basic setup is

$$
\begin{aligned}
& \theta_{i}=\theta_{i-1}+w_{i} \\
& y_{i}=\varphi_{i}^{T} \theta_{i}+v_{i}
\end{aligned}
$$

The typical algorithm has the form

$$
\hat{\theta}_{i}=\hat{\theta}_{i-1}+K_{i}\left(y_{i}-\varphi_{i}^{T} \hat{\theta}_{i-1}\right)
$$

Much effort has been spent on the choice of $K_{i}$ and the analysis of the tracking error $\tilde{\theta}_{i}=\theta_{i}-\hat{\theta}_{i}$. Note that under (2) this is a linear function of the driving disturbances and the initial condition:

$$
\tilde{\theta}_{i}=h_{i}^{t} \tilde{\theta}_{-1}+\sum_{j=0}^{i}\left(h_{j}^{v} v_{j}+h_{j}^{w} w_{j}\right)
$$

The coefficients $h_{j}$ are functions of $K_{\ell}$ and the regression vectors.

\footnotetext{
1 This work was supported in part by the Swedish Research Council for Engineering
}

We shall in this contribution review and expand on some so called $\mathcal{H}_{\infty}$ results of this kind. These are mainly drawn from the comprehensive papers (Hassibi et al., 1996b) and (Hassibi et al., 1996c).

First, let us however discuss the evaluation criterion. The basic idea is to view $\tilde{\theta}$ as the output of a system (3) driven by $v, w$ and the initial value $\tilde{\theta}_{-1}$. Consider the ratio

$$
\begin{aligned}
& V\left(K, \tilde{\theta}_{-1}, v, w\right)= \\
& =\frac{\sum_{i=0}^{N}\left|\tilde{\theta}_{i-1}\right|^{2}}{\left|\tilde{\theta}_{-1}\right|^{2} / \mu+\sum_{i=0}^{N}\left(\left|v_{i}\right|^{2} / R_{v}+\left|w_{i}\right|^{2} / R_{w}\right)}
\end{aligned}
$$

This can be seen as the gain of this system. The "worst case" sequences $v, w$ and initial value $\tilde{\theta}_{-1}$ will give an upper bound on this gain. We would then like to optimize the algorithm by choosing $K_{i}$ so that this upper bound is minimized:

$$
\min _{K_{i}} \max _{\tilde{\theta}_{-1}, v, w} V\left(K, \tilde{\theta}_{-1}, v, w\right)
$$

This gives the " $\mathcal{H}_{\infty}$-optimal" identification algorithm, and we shall in the following sections describe and discuss these solutions.

Two questions should be addressed first, though:

- Is (4) a reasonable criterion? 
- Is maximizing over worst case disturbances reasonable, or too pessimistic?

Let's start with the first one. What is special from an identification perspective is that the parameter errors at all time points are summed in the numerator. A typical behavior is that there is a considerable initial error and it's the algorithm's (i.e. the $K_{i}$ :s) duty to bring this error down quickly. It is then questionable why the initial errors should linger and typically dominate the numerator in the criterion. It may be more interesting to consider the alternative criterion at a given time point $N$ :

$$
\begin{aligned}
& V_{1}^{N}\left(K, \tilde{\theta}_{-1}, v, w\right)= \\
& =\frac{\left|\tilde{\theta}_{N-1}\right|^{2}}{\left|\theta_{-1}\right|^{2} / \mu+\sum_{i=0}^{N}\left(\left|v_{i}\right|^{2} / R_{v}+\left|w_{i}\right|^{2} / R_{w}\right)}
\end{aligned}
$$

To find the $K_{i}$ that solve the min-max problem (5) for $V_{1}^{N}$ is easy and known: Applying CauchySchwartz to (3) gives (we give the result for the case $\theta$ is scalar, for simplicity):

$$
\begin{aligned}
& \left|\tilde{\theta}_{N}\right|^{2} \leq\left[\mu\left(h_{N}^{t}\right)^{2}+\sum_{j=0}^{N}\left(R_{v}\left(h_{j}^{v}\right)^{2}+R_{w}\left(h_{j}^{w}\right)^{2}\right)\right] \times \\
& {\left[\left|\tilde{\theta}_{-1}\right|^{2} / \mu+\sum_{j=0}^{N}\left(w_{j}^{2} / R_{w}+v_{j}^{2} / R_{v}\right)\right]}
\end{aligned}
$$

where equality is achieved for certain sequences. Solving the min-max problem is thus the same as minimizing the expression within the first brackets. This expression is of course the variance of $\tilde{\theta}_{N}$ in case the inputs would be independent random variables with the indicated covariances. The minmax solution of $V_{1}$ thus coincides with the classical least squares/Kalman filter solutions. (In the language of $\mathcal{H}_{\infty}$, this corresponds to the fact that the $\mathcal{H}_{\infty}$ norm of a row vector is the same as the $\mathcal{H}_{2}$ norm.)

Moreover, it follows that the min-max bound on $V_{1}$ is the largest singular value of the $P$-matrix that solves the corresponding Riccati-equation.

We note in particular that the solution, i.e., the choice of $K_{i}$, does not depend on $N$. We could thus do "worst case" over $N$ and still obtain the same solution.

This result also sheds some light in the second question: Is it reasonable to work with worst case disturbances? It of course an attractive feature to make the analysis independent of probabilistic "noise" assumptions. In a sense this makes the results more robust. On the other hand, it is known from other contexts that designing an algorithm for the worst case might be very conservative. However, in the case of (6) these two aspects cooperate in a happy way: What is optimal in the stochastic setting is also optimally robust in a non-stochastic, worst case formulation.
In the following three sections we study the LMS, RLS and Kalman filter algorithms, respectively. First, in Section 2, we review some results on the $\mathcal{H}_{\infty}$ optimality of the LMS algorithm. Then, in Section 3, we give the corresponding results for the RLS algorithm. Section 4 contains a discussion on how the $\mathcal{H}_{\infty}$ filters may be seen as Kalman filters with special choices of the design variables, i.e., the covariance matrices. The main points of the paper are then summarized in Section 5.

\section{LMS IS $\mathcal{H}_{\infty}$ OPTIMAL}

Consider the time-invariant model $(w=0$ in $(1))$

$$
y_{i}=\varphi_{i}^{T} \theta+v_{i}
$$

The well-known LMS algorithm (see, e.g. (Widrow and Hoff Jr., 1960; Widrow and Stearns, 1985)) is defined by

$$
\hat{\theta}_{i}=\hat{\theta}_{i-1}+\mu \varphi_{i}\left(y_{i}-\varphi_{i}^{T} \hat{\theta}_{i-1}\right)
$$

LMS gives an approximate recursive solution to the following problem:

$$
\min _{\theta} V_{N}(\theta)=\min _{\theta} \sum_{i=0}^{N}\left|y_{i}-\varphi_{i}^{T} \theta\right|^{2}
$$

However, as shown in (Hassibi et al., 1996a), the following is also true: Given that the input data is exciting and that $0<\mu<\inf _{i} \frac{1}{\left|\varphi_{i}\right|^{2}}$, LMS guarantees

$$
\sup _{\tilde{\theta}_{-1}, v \in \ell_{2}} \frac{\|\varepsilon\|_{2}^{2}}{\frac{1}{\mu}\left\|\tilde{\theta}_{-1}\right\|^{2}+\|v\|_{2}^{2}}<1
$$

where $\varepsilon_{i}=\varphi_{i}^{T} \tilde{\theta}_{i-1}=\varphi_{i}^{T}\left(\theta-\hat{\theta}_{i-1}\right)$. Furthermore, the bound in (10) is optimal, i.e., LMS is $\mathcal{H}_{\infty}$ optimal. In (10) $\|\cdot\|_{2}$ denotes the $\ell_{2}$ norm:

$$
\|x\|_{2}=\left(\sum_{i=0}^{\infty}\left|x_{i}\right|^{2}\right)^{1 / 2}
$$

We will not go into any further details about the $\mathcal{H}_{\infty}$ filter results in (Hassibi et al., 1996a) at this point. Instead we will establish that LMS satisfies (10) using simple algebra. A similar derivation can be found in, e.g., (Sayed and Kailath, 1994). From (7) and (8) we have

$$
\tilde{\theta}_{i}=\theta-\hat{\theta}_{i}=\left(I-\mu \varphi_{i} \varphi_{i}^{T}\right) \tilde{\theta}_{i-1}-\mu \varphi_{i} v_{i}
$$

Squaring both sides gives, after some manipulations,

$$
\begin{aligned}
\left\|\tilde{\theta}_{i}\right\|^{2}= & \left\|\tilde{\theta}_{i-1}\right\|^{2}+\mu\left|v_{i}\right|^{2}-\mu\left|\varphi_{i}^{T} \tilde{\theta}_{i-1}\right|^{2} \\
& -\mu\left(1-\mu\left|\varphi_{i}\right|^{2}\right)\left|y_{i}-\varphi_{i}^{T} \hat{\theta}_{i-1}\right|^{2}
\end{aligned}
$$

Now, if $\mu<\inf _{i} \frac{1}{\sqrt{\left.\varphi_{i}\right|^{2}}}$, we get

$$
\left\|\tilde{\theta}_{i}\right\|^{2}+\mu\left|\varphi_{i}^{T} \tilde{\theta}_{i-1}\right|^{2}<\left\|\tilde{\theta}_{i-1}\right\|^{2}+\mu\left|v_{i}\right|^{2}
$$


Summing over $i=0, \ldots, N$ we thus have

$$
\left\|\tilde{\theta}_{N}\right\|^{2}+\mu \sum_{i=0}^{N}\left|\varphi_{i}^{T} \tilde{\theta}_{i-1}\right|^{2}<\left\|\tilde{\theta}_{-1}\right\|^{2}+\mu \sum_{i=0}^{N}\left|v_{i}\right|^{2}
$$

The inequality becomes even stricter if we ignore the first term on the left, hence we have shown

$$
\frac{\sum_{i=0}^{N}\left|\varphi_{i}^{T} \tilde{\theta}_{i-1}\right|^{2}}{\frac{1}{\mu}\left\|\tilde{\theta}_{-1}\right\|^{2}+\sum_{i=0}^{N}\left|v_{i}\right|^{2}}<1
$$

If we let $N \rightarrow \infty$ this proves (10) since $\tilde{\theta}_{-1}$ and $\left\{v_{i}\right\}$ was arbitrary in our derivation of (15).

\section{AN $\mathcal{H}_{\infty}$ BOUND FOR RLS}

Consider now the weighted least-squares criterion function

$$
V_{N}(\theta)=\sum_{i=0}^{N} \lambda^{N-i}\left(y_{i}-\varphi_{i}^{T} \theta\right)^{2}
$$

where $0 \ll \lambda<1$. The solution is

$$
\hat{\theta}_{N}=\left[\sum_{i=0}^{N} \lambda^{N-i} \varphi_{i} \varphi_{i}^{T}\right]^{-1} \sum_{i=0}^{N} \lambda^{N-i} \varphi_{i} y_{i}
$$

Define

$$
\bar{R}_{i}=\sum_{k=0}^{i} \lambda^{i-k} \varphi_{k} \varphi_{k}^{T}
$$

Then

$$
\bar{R}_{i}=\lambda \bar{R}_{i-1}+\varphi_{i} \varphi_{i}^{T}
$$

Inspecting (17) we see that this gives the following recursion for the estimate $\hat{\theta}_{i}$ :

$$
\hat{\theta}_{i}=\hat{\theta}_{i-1}+\bar{R}_{i}^{-1} \varphi_{i}\left(y_{i}-\varphi_{i}^{T} \hat{\theta}_{i-1}\right)
$$

Now introduce

$$
\bar{P}_{i}=\bar{R}_{i}^{-1}
$$

and apply the matrix inversion lemma to (19). This gives

$$
\bar{P}_{i}=\frac{1}{\lambda}\left(\bar{P}_{i-1}-\frac{\bar{P}_{i-1} \varphi_{i} \varphi_{i}^{T} \bar{P}_{i-1}}{\lambda+\varphi_{i}^{T} \bar{P}_{i-1} \varphi_{i}}\right)
$$

Since

$$
\bar{R}_{i}^{-1} \varphi_{i}=\bar{P}_{i} \varphi_{i}=\frac{\bar{P}_{i-1} \varphi_{i}}{\lambda+\varphi_{i}^{T} \bar{P}_{i-1} \varphi_{i}}
$$

we can rewrite (20) as

$$
\hat{\theta}_{i}=\hat{\theta}_{i-1}+\frac{\bar{P}_{i-1} \varphi_{i}}{\lambda+\varphi_{i}^{T} \bar{P}_{i-1} \varphi_{i}}\left(y_{i}-\varphi_{i}^{T} \hat{\theta}_{i-1}\right)
$$

Equations (24) and (22) are known as the recursive least squares (RLS) algorithm (see, e.g., (Ljung and Söderström, 1983) or (Haykin, 1996)).
Studying (17) we can easily derive the following expression for the error $\tilde{\theta}_{i}=\theta-\hat{\theta}_{i}$ :

$$
\tilde{\theta}_{i}=-\bar{R}_{i}^{-1} \sum_{k=0}^{i} \lambda^{i-k} \varphi_{k} v_{k}
$$

It will be convienient to rewrite this in vector notation:

$$
\tilde{\theta}_{i}=-\bar{R}_{i}^{-1} \Phi_{i} V_{i}
$$

with

$$
\begin{aligned}
\Phi_{i} & =\left[\lambda^{(N-1) / 2} \varphi_{1}, \ldots, \lambda^{1 / 2} \varphi_{i-1}, \varphi_{i}\right] \\
V_{i} & =\left[\lambda^{(N-1) / 2} v_{1}, \ldots, \lambda^{1 / 2} v_{i-1}, v_{i}\right]^{T}
\end{aligned}
$$

Now, since $\bar{R}_{i}=\Phi_{i} \Phi_{i}^{T}$, we get the following

$$
\begin{aligned}
\tilde{\theta}_{i}^{T} \bar{R}_{i} \tilde{\theta}_{i} & =V_{i}^{T} \Phi_{i}^{T} \bar{R}_{i}^{-1} \bar{R}_{i} \bar{R}_{i}^{-1} \Phi_{i} V_{i} \\
& =V_{i}^{T} \Phi_{i}^{T}\left(\Phi_{i} \Phi_{i}^{T}\right)^{-1} \Phi_{i} V_{i} \\
& \leq V_{i}^{T} V_{i}=\sum_{k=0}^{i} \lambda^{i-k} v_{k}^{2}
\end{aligned}
$$

Summing over all $i$ we get

$$
\begin{aligned}
\sum_{i=0}^{N} \tilde{\theta}_{i}^{T} \bar{R}_{i} \tilde{\theta}_{i} & \leq \sum_{i=0}^{N} \sum_{k=0}^{i} \lambda^{i-k} v_{k}^{2} \\
& =\sum_{k=0}^{N} v_{k}^{2} \sum_{i=k}^{N} \lambda^{i-k} \\
& =\frac{1-\lambda^{N-i+1}}{1-\lambda} \sum_{k=0}^{N} v_{k}^{2} \\
& \leq \frac{1}{1-\lambda} \sum_{i=0}^{N} v_{i}^{2}
\end{aligned}
$$

We have thus proven that RLS satisfies the following $\mathcal{H}_{\infty}$ bound

$$
\frac{\sum_{i=0}^{N} \tilde{\theta}_{i}^{T} \bar{R}_{i} \tilde{\theta}_{i}}{\sum_{i=0}^{N} v_{i}^{2}} \leq \frac{1}{1-\lambda}
$$

The bound (36) may in fact be tightened. Consider the following $\mathcal{H}_{\infty}$ result:

\section{Lemma 1. An estimator guaranteeing}

$$
\sup _{\theta_{0}, v \in \ell_{2}} \frac{\sum_{i=0}^{N} \tilde{\theta}^{T} \phi_{i} \phi_{i}^{T} \tilde{\theta}_{i}}{\tilde{\theta}_{0}^{T} \Pi_{0}^{-1} \tilde{\theta}_{0}+\frac{1}{\lambda} \sum_{i=0}^{N}\left|v_{i}\right|^{2}}<\gamma^{2}
$$

exists if, and only if,

$$
R_{i}>0, i=1, \ldots, N
$$

where $R_{i}$ obeys the recursion

$$
R_{i+1}=R_{i}-\gamma^{-2} \phi_{i} \phi_{i}^{T}+\frac{1}{\lambda} \varphi_{i} \varphi_{i}^{T}
$$

initialized with $R_{0}=\Pi_{0}^{-1}$. If this is the case, then the estimates can be computed as

$$
\hat{\theta}_{i}=\hat{\theta}_{i-1}+\frac{P_{i} \varphi_{i}}{\lambda+\varphi_{i}^{T} P_{i} \varphi_{i}}\left(y_{i}-\varphi_{i}^{T} \hat{\theta}_{i-1}\right)
$$

where $P_{i}=R_{i}^{-1}$. 
The recursion (19) can be rewritten as follows:

$$
\bar{R}_{i}=\bar{R}_{i-1}-(1-\lambda)\left(\bar{R}_{i-1}+\frac{1}{\lambda} \varphi_{i} \varphi_{i}^{T}\right)+\frac{1}{\lambda} \varphi_{i} \varphi_{i}^{T}
$$

This strongly resembles the recursion (39). Thus, if we make the following identifications,

$$
\begin{aligned}
R_{i+1} & =\bar{R}_{i}, P_{i+1}=\bar{P}_{i} \\
\gamma^{-2} & =1-\lambda \\
\phi_{i} \phi_{i}^{T} & =\bar{R}_{i-1}+\frac{1}{\lambda} \varphi_{i} \varphi_{i}^{T}=\frac{1}{\lambda} \bar{R}_{i}
\end{aligned}
$$

we conclude that:

Theorem 1. The RLS algorithm, with $\bar{P}_{i}$ initialized with $\bar{P}_{-1}=\Pi_{0}$, satisfies the $\mathcal{H}_{\infty}$ bound

$$
\sup _{\theta_{0}, v \in \ell_{2}} \frac{\sum_{i=0}^{N} \tilde{\theta}^{T} \bar{R}_{i} \tilde{\theta}_{i}}{\tilde{\theta}_{0}^{T} \Pi_{0}^{-1} \tilde{\theta}_{0}+\frac{1}{\lambda} \sum_{i=0}^{N}\left|v_{i}\right|^{2}}<\frac{\lambda}{1-\lambda}
$$

We note that, if $\Pi_{0} \rightarrow \infty$ this expression equals the bound (36) which was derived using simple algebra only. This result should be compared with the bounds derived in, e.g., (Hassibi and Kailath, 1994) and (Rupp and Sayed, 1995).

\section{LINKING $\mathcal{H}_{\infty}$ FILTERING TO KALMAN FILTERING}

Consider the random walk model (1) and the problem of recursively estimating the parameters $\theta_{i}$, given measurements of the output $y_{i}$. It is clear that we may use both the Kalman filter and the $\mathcal{H}_{\infty}$ filters to obtain estimates of $\theta_{i}$. The question is then whether our choice of algorithm matters. In this section we will try to answer this question, for instance by trying to relate the Kalman filter and the $\mathcal{H}_{\infty}$ filters through the Riccati recursion and the filter gains. The results in this section rely heavily on the $\mathcal{H}_{\infty}$ filtering results in (Hassibi et al., 1996b) and (Hassibi et al., 1996c).

\subsection{DISCRETE-TIME $\mathcal{H}_{\infty}$ FILTERS}

We now consider the case of $\mathcal{H}_{\infty}$ optimal filtering. Let $Q_{i}^{-1 / 2}$ and $R_{i}^{-1 / 2}$ be square-root factors of $Q_{i}$ and $R_{i}$, respectively. With

$$
\begin{aligned}
\bar{w}_{i} & =Q_{i}^{-1 / 2} w_{i}, i=0, \ldots, N \\
\bar{v}_{i} & =R_{i}^{-1 / 2} v_{i}, i=0, \ldots, N
\end{aligned}
$$

we can formulate the following sub-optimal $\mathcal{H}_{\infty}$ filtering problems:

(1) Given $\gamma>0$, find an estimation strategy that for all $\bar{w}, \bar{v} \in \ell_{2}[0, N-1]$ and all $\theta_{0}$ achieves the a priori bound

$$
\frac{\sum_{i=0}^{N}\left|\theta_{i}-\hat{\theta}_{i}\right|^{2}}{\tilde{\theta}_{0}^{T} \Pi_{0}^{-1} \tilde{\theta}_{0}+\sum_{i=0}^{N-1}\left|\bar{w}_{i}\right|^{2}+\sum_{i=0}^{N-1}\left|\bar{v}_{i}\right|^{2}}<\gamma^{2}
$$

(2) Given $\gamma>0$, find an estimation strategy that for all $\bar{w}, \bar{v} \in \ell_{2}[0, N]$ and all $\theta_{0}$ achieves the a posteriori bound

$$
\frac{\sum_{i=0}^{N}\left|\theta_{i}-\hat{\theta}_{i \mid i}\right|^{2}}{\tilde{\theta}_{0}^{T} \Pi_{0}^{-1} \tilde{\theta}_{0}+\sum_{i=0}^{N}\left|\bar{w}_{i}\right|^{2}+\sum_{i=0}^{N}\left|\bar{v}_{i}\right|^{2}}<\gamma^{2}
$$

We then have the following results which easily follow from the theorems in (Hassibi et al., 1996b) and (Hassibi et al., 1996c).

Corollary 1. (Sub-optimal $\mathcal{H}_{\infty}$ a priori filter) An estimator that achieves (46), for a given $\gamma>$ 0 , exists if, and only if,

$$
\tilde{P}_{i}^{-1}=P_{i}^{-1}-\gamma^{-2} I>0, i=0, \ldots, N
$$

where $P_{0}=\Pi_{0}$ and where $P_{i}$ obeys the Riccati recursion

$$
P_{i+1}=P_{i}+Q_{i}-P_{i}\left[\varphi_{i} I\right] R_{e, i}^{-1}\left[\begin{array}{c}
\varphi_{i}^{T} \\
I
\end{array}\right]
$$

with

$$
R_{e, i}=\left[\begin{array}{cc}
R_{i} & 0 \\
0 & -\gamma^{2} I
\end{array}\right]+\left[\begin{array}{c}
\varphi_{i}^{T} \\
I
\end{array}\right] P_{i}\left[\varphi_{i} I\right]
$$

If this is the case, then one possible level- $\gamma \mathcal{H}_{\infty}$ filter is given by

$$
\hat{\theta}_{i+1}=\hat{\theta}_{i}+K_{a, i}\left(y_{i}-\varphi_{i}^{T} \hat{\theta}_{i}\right)
$$

where

$$
K_{a, i}=\tilde{P}_{i} \varphi_{i}^{T}\left(R_{i}+\varphi_{i}^{T} \tilde{P}_{i} \varphi_{i}\right)^{-1}
$$

Corollary 2. (Sub-optimal $\mathcal{H}_{\infty}$ a posteriori filter) An estimator that achieves (47), for a given $\gamma>0$, exists if, and only if,

$$
P_{i}^{-1}+\varphi_{i}^{T} R_{i}^{-1} \varphi_{i}-\gamma^{-2} I>0, i=0, \ldots, N
$$

where $P_{i}$ is given by (49).

If this is the case, then one possible level- $\gamma \mathcal{H}_{\infty}$ a posteriori filter is given by

$$
\hat{\theta}_{i \mid i}=\hat{\theta}_{i-1 \mid i-1}+K_{s, i}\left(y_{i}-\varphi_{i}^{T} \hat{\theta}_{i-1 \mid i-1}\right)
$$

where

$$
K_{s, i}=P_{i} \varphi_{i}\left(R_{i}+\varphi_{i} P_{i} \varphi_{i}^{T}\right)^{-1}
$$

\subsection{THE DISCRETE-TIME KALMAN FILTER}

The well-known Kalman filter can also be formulated in a priori and a posteriori variants but with the model (1) these will coincide. The Kalman filter for the model (1) is given by

$$
\hat{\theta}_{i+1}=\hat{\theta}_{i}+K_{i}\left(y_{i}-\varphi_{i}^{T} \hat{\theta}_{i}\right)
$$

where the Kalman gain $K_{i}$ is given by

$$
K_{i}=P_{i} \varphi_{i} R_{e, i}^{-1}
$$

where

$$
R_{e, i}=R_{i}+\varphi_{i}^{T} P_{i} \varphi_{i}
$$


and where $P_{i}$ satisfies the following Riccati recursion

$$
P_{i+1}=P_{i}+Q_{i}-P_{i} \varphi_{i}\left(R_{i}+\varphi_{i}^{T} P_{i} \varphi_{i}\right)^{-1} \varphi_{i}^{T} P_{i}
$$

initialized with $P_{0}=\Pi_{0}$.

We may also use the following two-step procedure to update $P_{i}$, instead of (59)

$$
\left\{\begin{aligned}
P_{i \mid i} & =P_{i}-P_{i} \varphi_{i}\left(R_{i}+\varphi_{i}^{T} P_{i} \varphi_{i}\right)^{-1} \varphi_{i}^{T} P_{i}, \\
P_{i+1} & =P_{i \mid i}+Q_{i}
\end{aligned}\right.
$$

\subsection{KALMAN FILTER INTERPRETATION OF THE $\mathcal{H}_{\infty}$ FILTERS}

In this section we will show how the $\mathcal{H}_{\infty}$ filters can be seen as Kalman filters with particular choices of the design variables $Q_{i}$ and $R_{i}$. Similar results can be shown for the LMS and RLS algorithms (see, e.g., (Ljung and Gunnarsson, 1990)). A standing assumption in this section will be that $\gamma>0$ is such that (48) (or (53)) holds).

We will start with the $\mathcal{H}_{\infty}$ a posteriori filter since we, in that case, immediately can make the observation that

$$
K_{s, i}=P_{i} \varphi_{i}\left(R_{i}+\varphi_{i}^{T} P_{i} \varphi_{i}\right)^{-1}=K_{i}
$$

Hence the expressions for the $\mathcal{H}_{\infty}$ a posteriori filter gain and the Kalman gain are identical and, in particular, $R_{i}$ plays exactly in the same role in the two filters. To find out exactly what role $Q_{i}$ plays we may rewrite the Riccati recursion (49) as follows. Let

$$
P_{i \mid i}=P_{i}-P_{i} \varphi_{i}\left(R_{i}+\varphi_{i}^{T} P_{i} \varphi_{i}\right)^{-1} \varphi_{i}^{T} P_{i}
$$

and

$$
\Delta_{i}=P_{i \mid i}-\gamma^{2} I .
$$

Now, using Schur complements we can write

$$
\begin{gathered}
{\left[\begin{array}{cc}
R_{i}+\varphi_{i}^{T} P_{i} \varphi_{i} & \varphi_{i}^{T} P_{i} \\
P_{i} \varphi_{i} & P_{i}-\gamma^{2} I
\end{array}\right]^{-1}=\left[\begin{array}{cc}
1 & -K_{i}^{T} \\
0 & I
\end{array}\right]} \\
\cdot\left[\begin{array}{cc}
\left(R_{i}+\varphi_{i}^{T} P_{i} \varphi_{i}\right)^{-1} & 0 \\
0 & \Delta_{i}^{-1}
\end{array}\right]\left[\begin{array}{cc}
1 & 0 \\
-K_{i} & I
\end{array}\right]
\end{gathered}
$$

and, as can easily be verified, it follows that (49) can be rewritten as

$$
P_{i+1}=P_{i \mid i}+Q_{i}-P_{i \mid i}\left(P_{i \mid i}-\gamma^{2} I\right)^{-1} P_{i \mid i}
$$

Thus if we replace the covariance matrix $Q_{i}$ in the Kalman filter recursions with the quantity $Q_{i}+P_{i \mid i}\left(\gamma^{2} I-P_{i \mid i}\right)^{-1} P_{i \mid i}$ we re-obtain the $\mathcal{H}_{\infty}$ a posteriori filter equations. We may summarize the above calculations as follows.

Theorem 2. If we run the Kalman filter with $Q_{i}$ chosen as

$$
Q_{i}+P_{i \mid i}\left(\gamma^{2} I-P_{i \mid i}\right)^{-1} P_{i \mid i}
$$

the resulting filter satisfies the a posteriori bound (47) (given that $\gamma>0$ is large enough so that (47) holds).

Remark: If we rewrite the condition (53) as

$$
P_{i \mid i}^{-1}-\gamma^{-2} I>0, i=0, \ldots, N
$$

we see that

$$
P_{i \mid i}\left(\gamma^{2} I-P_{i \mid i}\right)^{-1} P_{i \mid i}>0, i=0, \ldots, N
$$

Changing $Q_{i}$ according to (66) can thus be seen as a raise of the covariance matrix $Q_{i}$ by the amount $P_{i \mid i}\left(\gamma^{2} I-P_{i \mid i}\right)^{-1} P_{i \mid i}$. We can therefore expect better tracking with this adjustment, compared to the standard Kalman filter.

In the a priori case a similar interpretation of the $\mathcal{H}_{\infty}$ filter will be less straight-forward and natural, but for completeness we will carry out the calculations anyhow. We start with the following result.

Theorem 3. The Kalman filter with $Q_{i}$ chosen as

$$
\left(\tilde{P}_{i \mid i}+Q_{i}\right)\left(I-\gamma^{-2}\left(\tilde{P}_{i \mid i}+Q_{i}\right)\right)^{-1}-\tilde{P}_{i \mid i}
$$

where

$$
\tilde{P}_{i \mid i}=\tilde{P}_{i}-\tilde{P}_{i} \varphi_{i}\left(R_{i}+\varphi_{i}^{T} \tilde{P}_{i} \varphi_{i}\right)^{-1} \varphi_{i}^{T} \tilde{P}_{i}
$$

and where $\tilde{P}_{i}$ as in Corollary 1, guarantees that the a priori bound (46) holds.

Proof: Studying Corollary 1 we see that the filter gain $K_{a, i}$ involves the quantity $\tilde{P}_{i}$. It is therefore natural to try to rewrite the Riccati recursion (49)) into an equivalent recursion involving $\tilde{P}_{i}$. Introducing

$$
\tilde{P}_{i \mid i}=P_{i}-P_{i}\left[\varphi_{i} I\right] R_{e, i}^{-1}\left[\begin{array}{c}
\varphi_{i}^{T} \\
I
\end{array}\right] P_{i}
$$

where $R_{e, i}$ is defined in (50), we see that (49) now can be written as

$$
P_{i+1}=\tilde{P}_{i \mid i}+Q_{i}
$$

However, using the matrix inversion lemma we also get

$$
\begin{aligned}
\tilde{P}_{i \mid i}^{-1} & =P_{i}^{-1}-\gamma^{-2} I+\varphi_{i} R_{i}^{-1} \varphi_{i}^{T} \\
& =\tilde{P}_{i}^{-1}+\varphi_{i} R_{i}^{-1} \varphi_{i}^{T}
\end{aligned}
$$

and we conclude, again using the matrix inversion lemma, that

$$
\tilde{P}_{i \mid i}=\tilde{P}_{i}-\tilde{P}_{i} \varphi_{i}\left(R_{i}+\varphi_{i}^{T} \tilde{P}_{i} \varphi_{i}\right)^{-1} \varphi_{i}^{T} \tilde{P}_{i}
$$

which is (69). Yet another application of the matrix inversion lemma shows that

$$
\tilde{P}_{i}=P_{i}-P_{i}\left(P_{i}-\gamma^{2} I\right)^{-1} P_{i}=P_{i}\left(I-\gamma^{-2} P_{i}\right)^{-1}
$$

Thus we have

$$
\tilde{P}_{i+1}=\left(\tilde{P}_{i \mid i}+Q_{i}\right)\left(I-\gamma^{-2}\left(\tilde{P}_{i \mid i}+Q_{i}\right)\right)^{-1}
$$

from which (68) follows, if we interpret $\tilde{P}_{i}$ as the error covariance matrix $P_{i}$ in, e.g., (59). 
From the calculations above we can also extract the following result.

Corollary 3. The Kalman filter with the following adjustment of the recursion for the matrix $P_{i}$ :

$$
\left\{\begin{aligned}
P_{i \mid i} & =P_{i}-P_{i} \varphi_{i}\left(R_{i}+\varphi_{i}^{T} P_{i} \varphi_{i}\right)^{-1} \varphi_{i}^{T} P_{i} \\
\bar{P}_{i+1} & =P_{i \mid i}+Q_{i} \\
P_{i+1} & =\bar{P}_{i+1}\left(I-\gamma^{-2} \bar{P}_{i+1}\right)^{-1}
\end{aligned}\right.
$$

guarantees (46).

We may note that condition (48) implies that for all $\gamma>0$,

$$
\tilde{P}_{i}>P_{i}
$$

Hence, the filter algorithm tries to ensure that the filter gain never vanishes. This extra raise of the $P$-matrix can also be interpreted as an "anti regularization" approach, as we shall see momentarily. To avoid covariance blow up when running the Kalman filter regularization of the following kind is often employed: According to Levenberg-Marquardt we should add a positive matrix $\delta I$ to our information matrix, i.e., to $P_{i}^{-1}$, to prevent the covariance matrix from growing without bounds. The adjusted information matrix becomes

$$
\check{P}_{i}^{-1}=P_{i}^{-1}+\delta I
$$

giving

$$
\check{P}_{i}=P_{i}\left(I+\delta P_{i}\right)^{-1}
$$

For all $\delta>0$,

$$
\check{P}_{i}<P_{i}
$$

Hence, $\mathcal{H}_{\infty}$ filtering and regularization have opposite effects. Loosely, in $\mathcal{H}_{\infty}$ filtering you increase the covariance matrices and when using regularization you make them smaller.

\section{CONCLUSIONS}

Linear tracking algorithms like (2) are frequently used also when stochastic assumptions about the disturbances may be questionable. It is therefore of clear interest to investigate their behavior for quite general sequences $v$ and $w$. We have in this paper commented on a few aspects of that kind. The basic points can be summarized as follows:

- Minimizing the worst case size of $\tilde{\theta}$ at any given time point (see (6), (5)) gives the classical Kalman filter/linear least squares solution to the tracking problem.

- Let $P$ be the solution to the Riccati equation (59). In a stochastic setting this matrix is the parameter error covariance matrix. In the worst case, the largest singular value of $P$ gives a tight upper bound for the ratio $V_{1}$ in (6). (Note however that the worst case is a factor $N$ "worse" than the averaged, stochastic case - The denominator of $V_{1}$ typically increases like $N$.)

- The two above facts are as such well known, and are shared by any linear least squares estimate.

- The $\mathcal{H}_{\infty}$ solution, (4), (5) can also be computed as a Kalman filter, but with modified covariance matrices. Essentially, these give a somewhat larger gain in the estimator.

- The relevance of the $\mathcal{H}_{\infty}$ criterion for identification applications can be questioned. The quality of the model at any given time may be more relevant than summing the model errors over time, thus giving possibly large initial errors a substantial influence.

\section{REFERENCES}

Hassibi, B., A. H. Sayed and T. Kailath (1996a). $H^{\infty}$ Optimality of the LMS Algorithm. IEEE Transactions on Signal Processing 44(2), 267-280.

Hassibi, B., A. H. Sayed and T. Kailath (1996b). Linear Estimation in Krein Spaces - Part I: Theory. IEEE Transactions on Automatic Control 41(1), 18-33.

Hassibi, B., A. H. Sayed and T. Kailath (1996c). Linear Estimation in Krein Spaces - Part II: Applications. IEEE Transactions on Automatic Control 41(1), 33-49.

Hassibi, B. and T. Kailath (1994). $H^{\infty}$ Bounds for the Recursive-Least-Squares Algorithm. In: Proceedings of the 31st Conference on Decision and Control. Orlando, FL. pp. 39273929.

Haykin, S. (1996). Adaptive Filter Theory. 3rd ed.. Prentice-Hall.

Ljung, L. and S. Gunnarsson (1990). Adaptation and Tracking in System Identification - A Survey. Automatica 26(1), 7-21.

Ljung, L. and T. Söderström (1983). Theory and Practice of Recursive Identification. MIT Press.

Rupp, M. and A. H. Sayed (1995). Local and global passivity relations for Gauss-Newton methods in adaptive filtering. In: Proceedings of the SPIE Conference on Adv. Signal Processing. San Diego, CA.

Sayed, A. H. and T. Kailath (1994). A State-Space Approach to Adaptive RLS Filtering. IEEE Signal Processing Magazine 11(3), 18-60.

Widrow, B. and M. E. Hoff Jr. (1960). Adaptive switching circuits. In: IRE WESCON Convention Record. Vol. 4. pp. 96-104.

Widrow, B. and S. Stearns (1985). Adaptive Signal Processing. Prentice-Hall. Englewood Cliffs, N.J. 